\title{
Von Kries Model under Planckian Illuminants
}

\author{
Michela Lecca and Stefano Messelodi \\ Fondazione Bruno Kessler - Center for Information Technology \\ via Sommarive 18, Povo, 38123 Trento, Italy \\ \{lecca,messelod\}@fbk.eu
}

\begin{abstract}
Planckian illuminants and von Kries diagonal model are commonly assumed by many computer vision algorithms for modeling the color variations between two images of a same scene captured under two different illuminants. Here we present a method to estimate a von Kries transform approximating a Planckian illuminant change and we show that the Planckian assumption constraints the von Kries coefficients to belong to a ruled surface, that depends on physical cues of the lights. Moreover, we provide an approximated parametric representation of such a surface, making evident the dependence of the von Kries transform on the light color temperature and on the intensity.
\end{abstract}

\section{Introduction}

A same scene captured under two different lights produces different colors. This is because the color of an image recorded by a camera depends on the spectral power of the light illuminating the scene, on the geometrical and physical conditions of the scene and on the characteristics of the device used for the acquisition. Understanding the relation between the colors of two scenes imaged under different light is a crucial task for its many applications, like image retrieval and indexing [18, image segmentation [16], and shadow removal [2].

The von Kries diagonal model and/or Planckian illuminants are two common assumptions for modeling the color variations due to light changes. In this paper we investigate the relation between these two hypotheses.

The von Kries model approximates an illuminant change by a linear diagonal map that rescales independently the camera responses. The diagonal elements of the matrix representing this map are named von Kries coefficients and they completely determine the color transform. Despite its simplicity, the von Kries model has been proved to be a good approximation for color variations due to a photometric change [4], 5], 1].

Planckian illuminants are lights whose spectral power can be expressed by Planck's law, i.e. they behave like a black body radiator. Many natural lights such as the sunlight and fluorescent lamps satisfy Planck's approximation. This hypothesis is used in many recent works for removing shadows from pictures [8], [7. Changes of Planckian illuminants are commonly modeled by a linear map, called Bradford transform and defined on the XYZ color space [11, [20]. 
The contributions of our work are three:

1. given a Bradford transform $\tau$ relating two Planckian illuminants, we estimate its diagonal approximation $\mathcal{K}$ by a least square based method, that computes the von Kries coefficients of $\mathcal{K}$ by minimizing a $L^{1}$ distance from images reilluminated by $\tau$ and $\mathcal{K}$; according to the work [4], we empirically show that a linear diagonal map suffices for describing a Planckian illuminant change;

2. we show that under the Planckian assumption the von Kries coefficients are not independent each to other but they form a ruled surface parametrized by the physical cues of the varying illuminants;

3. finally, we derive an approximated equation for the von Kries surface and we show how it can be used for computing the color temperature and intensity of an illuminant.

Our empirical analysis has been carried out on two public real-world datasets [15, 3] and on some synthetic data generated from them.

Section 2 describes the von Kries model; Section 3 defines the Planckian lights and illustrates the Bradford model. Section 4 reports our experimental analysis and conclusions.

\section{The von Kries Model}

Hereafter we assume that the response of a camera to the light reflected from a point $x$ in a scene is coded in a triplet $\mathbf{p}^{T}(x)=\left(p_{0}(x), p_{1}(x), p_{2}(x)\right)$, where

$$
p_{i}(x)=\int_{\Omega} E(\lambda) S(\lambda, x) F_{i}(\lambda) d \lambda, \quad i=0,1,2 .
$$

$\lambda$ is the wavelength of the light illuminating the scene, $E$ its spectral power distribution, $S$ the reflectance of the surface to which the point belongs, and $F_{i}$ is the spectral sensitivity function of the sensor. The integral ranges over the visible spectrum, i.e. $\Omega=[380,780] \mathrm{nm}$.

The von Kries diagonal model approximates the spectral sensitivity of the camera sensor by a delta function, i.e. it assumes that each sensor responds only to a single wavelength of light: $F_{i}(\lambda)=\delta\left(\lambda-\lambda_{i}\right)$, for each $i=0,1,2$. With this assumption, the responses $\mathbf{p}(x)$ and $\mathbf{p}(x)$ at $x$ taken by the same camera under two different illuminants are linearly related by the following Equation:

$$
\left(p_{0}(x), p_{1}(x), p_{2}(x)\right)=\left(\frac{E\left(\lambda_{0}\right)}{E^{\prime}\left(\lambda_{0}\right)} p_{0}^{\prime}(x), \frac{E\left(\lambda_{1}\right)}{E^{\prime}\left(\lambda_{1}\right)} p_{1}^{\prime}(x), \frac{E\left(\lambda_{2}\right)}{E^{\prime}\left(\lambda_{2}\right)} p_{2}^{\prime}(x)\right)
$$

i.e. the von Kries diagonal model approximates the change of illuminant mapping $\mathbf{p}$ onto $\mathbf{p}^{\prime}$ by a linear transform that rescales each channel independently.

In the following, for each $i=0,1,2$, we set $\alpha_{i}:=E\left(\lambda_{i}\right)\left[E^{\prime}\left(\lambda_{i}\right)\right]^{-1}$ and we refer to the parameters $\alpha_{0}, \alpha_{1}$ and $\alpha_{2}$ as the von Kries coefficients. 


\section{Planckian Illuminants and Bradford Transform}

An illuminant is said Planckian if its spectral power is given by the Planck law

$$
E(\lambda, T, I)=I c_{1} \lambda^{-5}\left(e^{\frac{c_{2}}{T \lambda}}-1\right)^{-1}
$$

In this Equation, variables $\lambda, I$ and $T$ denote respectively the wavelength, the intensity and the color temperature of the illuminant, while the terms $c_{1}$ and $c_{2}$ are constants, more precisely $c_{1}=3.74183 \cdot 10^{-16} \mathrm{~W} \mathrm{~m} \mathrm{~m}^{2}$ and $c_{2}=1.4388 \cdot 10^{-2}$ $\mathrm{K} \mathrm{m}(\mathrm{W}=$ Watt, $\mathrm{m}=$ meter, $\mathrm{K}=$ Degree Kelvin). The intensity of the light describes its brightness and the color temperature is a measurement in Degrees Kelvin of its hue. For instance, the color temperature of a candle flame ranges over $[1850,1930] \mathrm{K}$, while the sun light at sunrise or at sunset has a color temperature between 2000 and $3000 \mathrm{~K}$.

The color of a Planckian light is often codified by the $2 \mathrm{D}$ vector $\chi$ of its chromaticities in the CIE XYZ color space. The computation of the color temperature of a light source is not easy because it differs from light to light upon the physical nature of the light. The chromaticities of the most Planckian illuminants have been tabulated empirically [10, 9]. Approximated formulas are also available [14].

A color change due to a variation of Planckian illuminants is modeled by the Bradford transform [11], [20]. This relates the XYZ coordinates $[X, Y, Z]$ and $\left[X^{\prime}, Y^{\prime}, Z^{\prime}\right]$ of the RGB responses $\mathbf{p}$ and $\mathbf{p}^{\prime}$ by the linear transform

$$
\left[X^{\prime}, Y^{\prime}, Z^{\prime}\right]^{T}=M \mathcal{D} M^{-1}[X, Y, Z]^{T},
$$

where $M$ is the Bradford matrix and $\mathcal{D}$ is a diagonal matrix representing the relation between the colorimetric properties (color temperatures and intensities) of $\sigma$ and $\sigma^{\prime}$.

Bradford matrix has been obtained empirically from Lam's experiments described in [1]:

$$
M=\left[\begin{array}{ccc}
0.8951 & 0.2664 & -0.1614 \\
-0.7502 & 1.7135 & 0.0367 \\
0.0389 & -0.0685 & 1.0296
\end{array}\right] \text { and } \mathcal{D}=\frac{Y_{\sigma}}{Y_{\sigma^{\prime}}}\left[\begin{array}{cccc}
\frac{x_{\sigma}}{y_{\sigma}} \frac{y_{\sigma^{\prime}}}{x_{\sigma^{\prime}}} & 0 & 0 \\
0 & 1 & 0 \\
0 & 0 \frac{1-x_{\sigma}-y_{\sigma}}{y_{\sigma}} \frac{y_{\sigma^{\prime}}}{1-x_{\sigma^{\prime}}-y_{\sigma^{\prime}}}
\end{array}\right]
$$

where $\left[x_{\sigma}, y_{\sigma}\right]$ and $\left[x_{\sigma^{\prime}}, y_{\sigma^{\prime}}\right]$ are the chromaticities of the color temperatures of $\sigma$ and $\sigma^{\prime}$ respectively. $Y_{\sigma}$ and $Y_{\sigma^{\prime}}$ are the $Y$ coordinates of the white reference of the illuminants $\sigma$ and $\sigma^{\prime}$ respectively.

In the RGB space, the Bradford transform can be re-written as follows:

$$
\mathbf{p}^{\prime T}=C M \mathcal{D} M^{-1} C^{-1} \mathbf{p}^{T}:=B \mathbf{p}^{T}
$$

where $C$ is the $3 \times 3$ matrix mapping the $\mathrm{XYZ}$ coordinates into the RGB coordinates and $B$ indicates the product $C M \mathcal{D} M^{-1} C^{-1}$. 


\section{Von Kries Coefficients under Planckian Illuminants}

Here we analyze the relation between the von Kries model and the Planckian assumption. The Section is organized as follows. In Subsection 4.1 we provide a method for approximating a Planckian illuminant change by a von Kries transform. In Subsection 4.2 we measure the goodness of the proposed approximation. In Subsection 4.3 we show how the von Kries coefficients are constrained by Planckian illuminants and how this fact can be used for determining the physical cues of the illuminant of an image. Finally, in Subsection 4.4, we summarize the results we obtained and we outlines our future work.

The experiments we describe here, have been carried out on the public databases Outex [15] and UEA dataset [3]. Both these databases consist of images taken under three different Planckian illuminants.

The Outex database collects different image sets for empirical evaluation of texture classification and segmentation algorithms. In this work we consider the test set named Outex_TC_00014, which consists of three sets of 1360 texture images viewed under the illuminants INCA, TL84 and HORIZON with color temperature $2856 \mathrm{~K}, 4100 \mathrm{~K}$ and $2300 \mathrm{~K}$ respectively. Figure 1] shows an image of Outex taken under these illuminants.
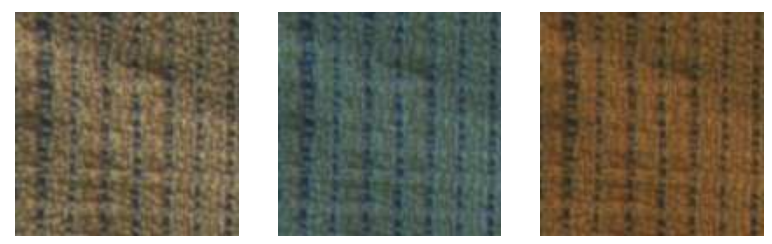

Fig. 1. Outex: a texture image taken under the Planckian illuminants INCA, TL84 and HORIZON (from left to right)

The UEA dataset comprises 28 design patterns, each one captured under 3 illuminants with 4 different cameras. The illuminants are indicated by Ill A (tungsten filament light, $2856 \mathrm{~K}$ ), Ill D65 (simulated daylight, $6500 \mathrm{~K}$ ), and TL84 (fluorescent tube, $4100 \mathrm{~K}$ ). An example is shown in Figure 2 .
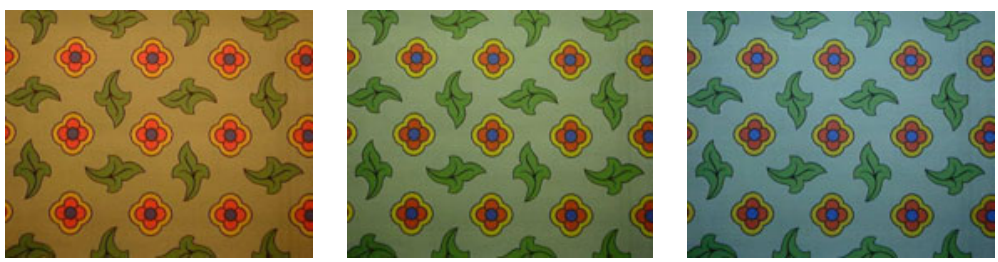

Fig. 2. UEA dataset: an image taken by Camera 1 under the Planckian illuminants Ill A, TL84 and Ill D65 (from left to right) 


\subsection{Estimating the von Kries Approximation}

The algorithm we propose for estimating the von Kries approximation does not consider possible affine distortions between the re-illuminated images, like for instance change of scales or of in-plane orientation. For affine transformed images, other methods can be used, as for instance [12, [13].

Given an image $\mathcal{A}$ taken under a source illuminant $\sigma$ and a Planckian illuminant change $\tau$ modeled by the Bradford transform (5), we define the von Kries approximation $\mathcal{K}$ of $\tau$ as the diagonal linear map whose coefficients $\alpha_{0}, \alpha_{1}, \alpha_{2}$ minimize the following distance:

$$
d\left(\alpha_{0}, \alpha_{1}, \alpha_{2}\right):=\sum_{x \in \mathcal{A}}\left\|(B-K) \mathbf{p}(x)^{T}\right\|^{2} .
$$

Here $B$ and $K$ are the matrices associated (with respect to the canonical basis of $\mathbf{R}^{3}$ ) to $\tau$ and $\mathcal{K}$ respectively. The $i$ th diagonal element $K_{i i}$ of $K$ is the $i$ th von Kries coefficient $\alpha_{i}$. The sum is computed over all the pixels $x$ of the image $\mathcal{A}$.

The $i$ th von Kries coefficient minimizing (6) is thus given by

$$
\alpha_{i}=\frac{\sum_{x} \sum_{j=0}^{2} b_{i j} p_{i}(x) p_{j}(x)}{\sum_{x} p_{i}(x)^{2}}
$$

Hereafter we indicate by $\tau(\mathcal{A})$ and $\mathcal{K}(\mathcal{A})$ the images obtained by re-illuminating $\mathcal{A}$ by $\tau$ and $\mathcal{K}$ respectively.

(a)

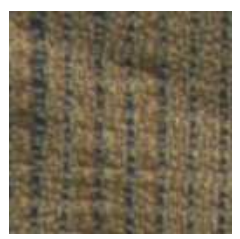

(b)

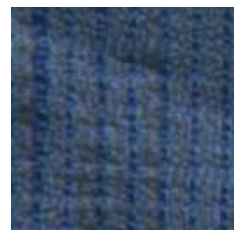

(c)

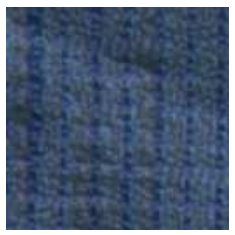

Fig. 3. Outex: The image (a) has been re-illuminated by a transform $\tau$ mapping the color temperature of INCA $(2856 \mathrm{~K})$ onto $6500 \mathrm{~K}$ and rescaling its intensity by 0.5 . The result is the picture (b). Image (c) is the image (a) remapped by our estimate $\mathcal{K}$. Images (b) and (c) look very similar.

\subsection{Evaluation of the von Kries Approximation}

For each source illuminant $\sigma \in \mathcal{I}=\{$ INCA/ILL A, TL84, HORIZON/ILL D65 $\}$ of the datasets Outex and UEA, we defined a set $\mathcal{T}$ of 40 Bradford transforms (Equation (5)), mapping $\sigma$ onto an other Planckian illuminant $\sigma^{\prime}$, with color temperature $T_{\sigma^{\prime}}=(2000+1000 t) \mathrm{K}$ and intensity $I_{\sigma^{\prime}}=(0.5+0.25 \mathrm{~m})$ with $t=$ $0, \ldots, 8, m=0, \ldots, 4$. For the considered datasets, no information about the intensity of the source illuminants are provided. Therefore, each transform of $\mathcal{T}$ rescales the intensity $I_{\sigma}$ of the source illuminant by $0.5,0.75,1.0,1.25$ and 1.5 . Figure 3 shows an example of such a transform. 
We re-illuminated each image of Outex and UEA captured under the illuminant $\sigma$ by these transforms and for each of them we estimate the von Kries approximation.

First of all, we note that the von Kries coefficients $\alpha_{0}, \alpha_{1}$ and $\alpha_{2}$ do not depend on the image $\mathcal{A}$ used in Equation (6). In fact, let $\alpha_{i}^{\mathcal{A}}, i=0,1,2$, be the von Kries coefficients of the transform $\mathcal{K}$ approximating $\tau$ for the image $\mathcal{A}$. Indicated by $N_{D B}$ the number of images in the database viewed under the illuminant $\sigma$, we computed the mean values

$$
\alpha_{i}=\frac{1}{N_{D B}} \sum_{\mathcal{A}} \alpha_{i}^{\mathcal{A}}, \quad i=0,1,2 .
$$

and we observed that the standard deviations from these values are very small, being them 0.03 on average, that is the $3 \%$ of the measure.

For each pair of images $(\tau(\mathcal{A}), \mathcal{K}(\mathcal{A}))$, we measure the goodness of the estimate of $\mathcal{K}$ on $\mathcal{A}$ by the $L^{1}$ RGB distance between the images $\tau(\mathcal{A})$ and $\mathcal{K}(\mathcal{A})$ :

$$
d_{\tau(\mathcal{A}), \mathcal{K}(\mathcal{A})}=\frac{1}{N} \sum_{x}\left|\mathbf{p}(x)^{\tau(\mathcal{A})}-\mathbf{p}(x)^{\mathcal{K}(\mathcal{A})}\right|,
$$

where $N$ is the number of pixels of $\mathcal{A}$. Distance (9) has been normalized to range over $[0,1]$. Closer to zero $d_{\tau, \mathcal{K}}$ is, more accurate the von Kries approximation is. Then, we measure the accuracy of the approximation $\mathcal{K}$ by the mean value $d_{\tau, \mathcal{K}}$ of $d_{\tau(\mathcal{A}), \mathcal{K}(\mathcal{A})}$ averaged on the number of database images (1360 for Outex, 28 for each camera of UEA). To measure how much a transform $\tau$ modifies the image, we compute the RGB distance $d_{\mathcal{A}, \mathcal{A}^{\prime}}$ : we expected that the mean value of $d_{\mathcal{A}, \mathcal{A}^{\prime}}$ is much greater than $d_{\tau, \mathcal{K}}$.

Figure 4 shows the results for Outex database, for which the accuracy $d_{\tau, \mathcal{K}}$ varies from 0.0 to 0.057 and is 0.022 on average. Similar results are obtained for UEA, where the mean value of $d_{\mathcal{A}, \mathcal{A}^{\prime}}$ varies from 0.002 to 0.052 and its average is 0.014 . The value of the mean RGB distance $d_{\mathcal{A}, \mathcal{A}^{\prime}}$ ranges over $[0.005,0.333]$ with average 0.177 for Outex, and over $[0.012,0.284]$ with average 0.176 for UEA.

According to 4, we found that the von Kries model is a good approximation for a Planckian illuminant change. However, the approximation accuracy decreases by increasing the intensity and the color temperature of the illuminant, i.e. by increasing the number of saturated pixels, whose percentage is particularly high (greater than 50\%) for the transforms with a color temperature gap of $4000 \mathrm{~K}$ and intensity 1.25 times greater than that of the original image. Figure 3 shows an example of color correction provided by our estimate. We got analogous accuracies for the estimate of the von Kries map that approximates the changes between two illuminants $\sigma$ and $\sigma^{\prime}$ of $\mathcal{I}$ on the real-world images of Outex and UEA.

\subsection{Constraints on the von Kries Coefficients}

Our experiments show that the von Kries coefficients we estimated are not independent each to other. We claim that the Planckian assumption constraints the 

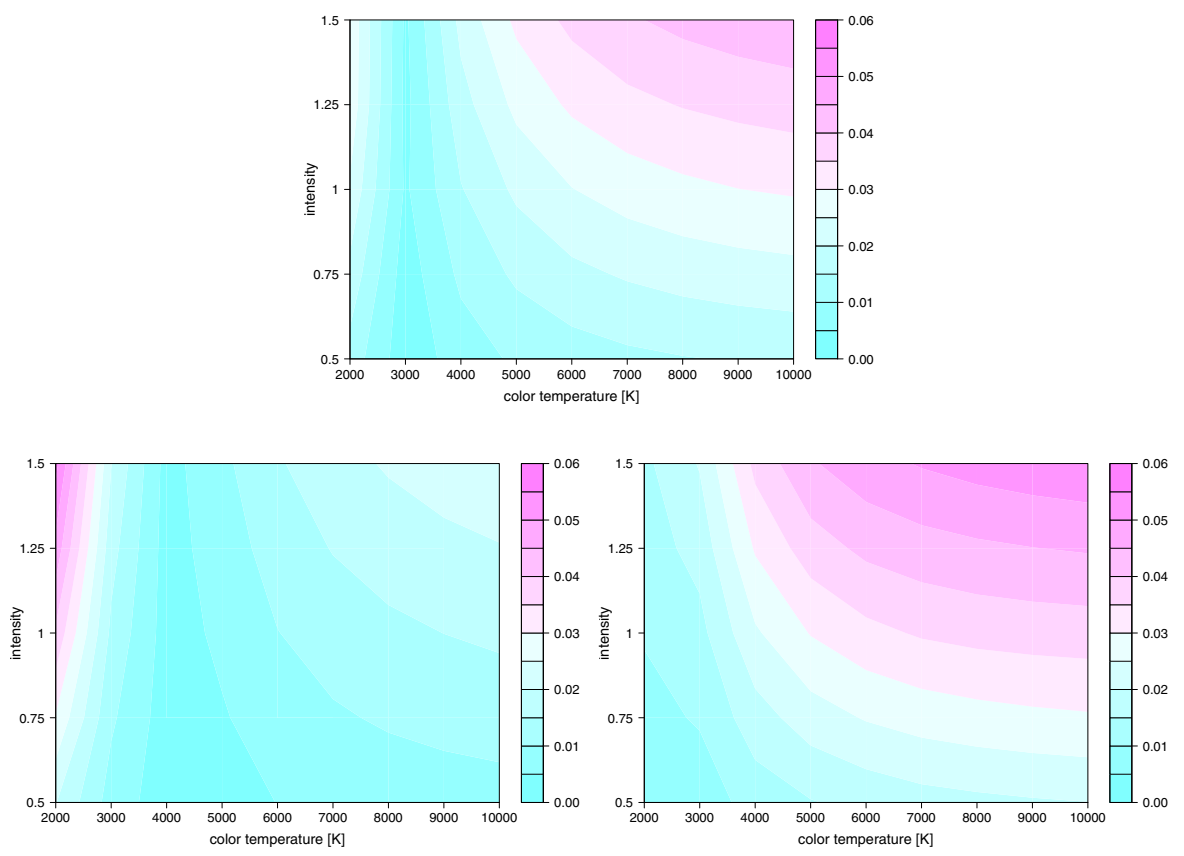

Fig. 4. Outex: INCA (top), TL84 (bottom, left), and HORIZON (bottom, right). $L^{1}$ RGB distance between the images re-illuminated by the Planckian model and the images re-illuminated by our von Kries approximation. The $L^{1} \mathrm{RGB}$ distance varies from 0.0 to 0.06 .

triplets $\left(\alpha_{0}, \alpha_{1}, \alpha_{2}\right)$ to belong to a ruled surface $\mathcal{S}$ (that we name von Kries surface) dependent on the color temperatures and on the variation of the illuminant intensities.

Each source illuminant $\sigma$ with color temperature $T_{\sigma}$ and intensity $I_{\sigma}$ defines a von Kries surface $\mathcal{S}$ given by

$$
\alpha_{i}=\alpha_{i}\left(T_{\sigma^{\prime}}, I_{\sigma^{\prime}}\right) \quad i=0,1,2,
$$

where the $\alpha_{i}$ 's are the von Kries coefficients of the map $\mathcal{K}$ that approximates the change moving $\sigma$ onto a Planckian illuminant $\sigma^{\prime}$ with color temperature $T_{\sigma^{\prime}}$ and intensity $I_{\sigma^{\prime}}$.

From Equations (5) and (7), we have that each von Kries coefficient can be re-written as $\alpha_{i}=\frac{Y_{\sigma}}{Y_{\sigma^{\prime}}} \alpha_{i}^{*}\left(T_{\sigma^{\prime}}\right)$, where $\alpha_{i}^{*}$ is the $i$ th von Kries coefficient of the linear map $\mathcal{K}$ that approximates the Bradford transform $\tau^{*}$ mapping $T_{\sigma}$ onto $T_{\sigma^{\prime}}$ and leaving unchanged the light intensity $I_{\sigma}$. By observing that $\frac{Y_{\sigma}}{Y_{\sigma^{\prime}}}=\frac{I_{\sigma}}{I_{\sigma^{\prime}}}$, we have that $\alpha_{i}=\frac{I_{\sigma}}{I_{\sigma^{\prime}}} \alpha_{i}^{*}\left(T_{\sigma^{\prime}}\right)$. Let $K$ and $B^{*}$ be the matrices representing $\mathcal{K}$ and $\tau^{*}$ in the canonical basis of $\mathbf{R}^{3}$. Since $\mathcal{K}$ is an approximation of $\tau^{*}$, there exists a matrix $H$ such that $K=H B$ and the difference $K-H B$ is close to the 

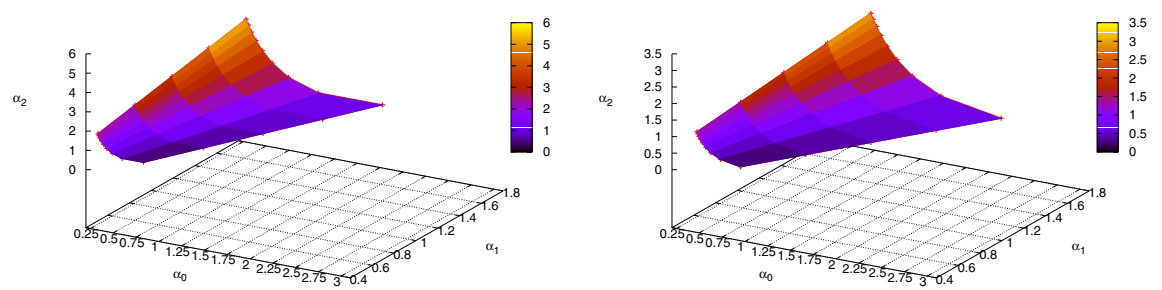

Fig. 5. UEA dataset: von Kries surfaces of cameras 1 (top) and 4 (bottom) for the illuminant $\sigma=$ ILL A

identity matrix. Therefore, $\alpha_{i}^{*}\left(T_{\sigma^{\prime}}\right)=\sum_{j=0}^{2} h_{i j} b_{j i}^{*}\left(T_{\sigma^{\prime}}\right)$, where $i=0,1,2$, and $h_{i j}$ is the $i j$-th element of $H$ and $b_{j i}^{*}$ is the $i j$-th element of $B^{*}$. We have that

$$
\alpha_{i}\left(I_{\sigma^{\prime}}, T_{\sigma^{\prime}}\right)=\frac{I_{\sigma}}{I_{\sigma^{\prime}}} \sum_{j=0}^{2} h_{i j} b_{i i}^{*}\left(T_{\sigma^{\prime}}\right) .
$$

Equation (11) makes evident the relation between the von Kries coefficients and the photometric properties of the illuminants $\sigma$ and $\sigma^{\prime}$ : it describes a ruled surface depending on the intensity and on the color temperature of $\sigma^{\prime}$. By varying discretely the intensity and the color temperature of the source illuminant $\sigma$, we obtain a sheaves of such surfaces.

As a consequence, we have that von Kries surface $\mathcal{S}$ is completely determined by the Equation of the $3 \mathrm{D}$ curve $\alpha^{*}\left(T_{\sigma}^{\prime}\right)=\left(\alpha_{0}^{*}\left(T_{\sigma}^{\prime}\right), \alpha_{1}^{*}\left(T_{\sigma}^{\prime}\right), \alpha_{2}^{*}\left(T_{\sigma}^{\prime}\right)\right)$, because each point on $\mathcal{S}$ is a rescaled version of a point onto $\alpha^{*}\left(T_{\sigma}^{\prime}\right)$. Thus, the von Kries maps that approximates Planckian variations of the color temperature but not of the intensity suffices for determining the von Kries surface.

We remark that for each fixed source illuminant $\sigma$ there exists a von Kries surface, that differs from device to device. This is because the values of the von Kries coefficients depend on the acquisition device (see for instance, Figure 5).

Finally, we briefly discuss a possible usage of the von Kries surfaces for recovering the color temperature and the intensity of an illuminant, a crucial task for many imaging applications [17], 19].

Let $\sigma$ be a Planckian illuminant with known color temperature $T_{\sigma}$ and intensity $I_{\sigma}$ and let $\mathcal{S}$ the von Kries surface of a camera $C$ computed with respect to the source illuminant $\sigma$. Let $\mathcal{A}$ and $\mathcal{A}^{\prime}$ be two images of the same scene taken by $C$ under the illuminants $\sigma$ and $\sigma^{\prime}$ respectively. Of course we suppose $\sigma$ and $\sigma^{\prime}$ to be Planckian. We employ the von Kries surface $\mathcal{S}$ to determine the color temperature $T_{\sigma^{\prime}}$ and the intensity $I_{\sigma^{\prime}}$ of $\sigma^{\prime}$ as follows: (1) we estimate the von Kries coefficients $\beta_{0}, \beta_{1}, \beta_{2}$ of the von Kries map relating $\mathcal{A}$ and $\mathcal{A}^{\prime} ;(2)$ we compute the triplet $\left(\alpha_{0}, \alpha_{1}, \alpha_{2}\right)$ on the von Kries map that has the minimum Euclidean distance from $\left(\beta_{0}, \beta_{1}, \beta_{2}\right)$ and we return the color temperature and intensity correspondent to $\left(\alpha_{0}, \alpha_{1}, \alpha_{2}\right)$. 
Here we report the results obtained on the database Outex for the source illuminant TL84. In this case, we considered the image pairs $\left(\mathcal{A}, \mathcal{A}^{\prime}\right)$ where $\mathcal{A}$ is an Outex picture imaged under TL84 and $\mathcal{A}^{\prime}$ is the same picture under INCA or HORIZON. We choose this case among the others because for it we got the most accurate estimates of the von Kries coefficients. Figures 6) (a) and (b) show the von Kries surface $\mathcal{S}$ for TL84 with the estimates of the von Kries coefficients of the transforms mapping (a) TL84 onto INCA and (b) TL84 onto HORIZON, respectively. The estimates we obtain determine a range of color temperatures and intensities. In particular, the color temperature of INCA varies over [3000, $4000] \mathrm{K}$, but the $70 \%$ about of the estimates are closer to $3000 \mathrm{~K}$ than to $4000 \mathrm{~K}$. For HORIZON the color temperature ranges over [2000, 4000] K with the most part of the data (about the $90 \%$ ) in [2000 K, 3000 K]. Similarly, we obtained a variability range for the intensity, with the $99 \%$ of the estimates between 1.0 and 1.25 for INCA and between 0.75 and 1.0 for HORIZON.

The results can be further refined, by considering a finer von Kries surface and by restricting the search for the triplet realizing the minimum distance to the ranges found before. Nevertheless, in general, obtaining an accurate estimate of these photometric parameters is a hard problem [19], also when calibrated images are used [6].

\subsection{Conclusions and Future Directions}

In this work we show empirically that the von Kries model approximates well a change of Planck's illuminants. The main consequence is that under Planck's hypothesis, the von Kries coefficients are the points of a ruled surface, whose mathematical expression highlights their dependency on the physical properties of the light and on the camera cues. Here we used the von Kries surfaces for estimating the color temperature and intensity of the illuminant under which an image has been captured. Our future work will be focus on other possible applications of this results, as for instance the development of device-independent approaches for the estimation of scene illuminants.
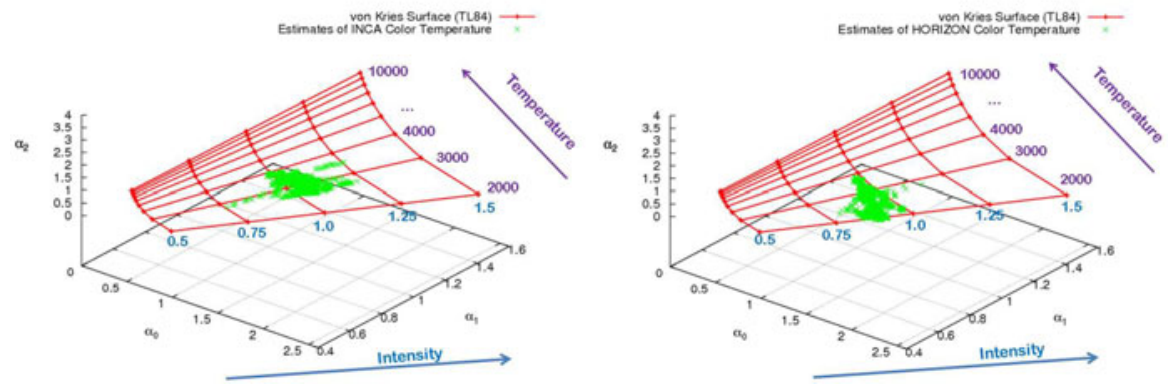

Fig. 6. Outex: color temperature and intensity estimates for illuminant INCA (on left) and HORIZON (on right) by using the von Kries surface with respect to TL84 


\section{References}

1. Barnard, K., Ciurea, F., Funt, B.: Sensor sharpening for computational color constancy. J. Opt. Soc. Am. A 18, 2728-2743 (2001)

2. Finlayson, G.D., Hordley, S.D., Drew, M.S.: Removing shadows from images. In: Heyden, A., Sparr, G., Nielsen, M., Johansen, P. (eds.) ECCV 2002. LNCS, vol. 2353, pp. 129-132. Springer, Heidelberg (2002)

3. Finlayson, G., Schaefer, G., Tian, G.Y.: The UEA uncalibrated colour image database. Technical Report SYS-C00-07, School of Information Systems, University of East Anglia, Norwich, United Kingdom (2000)

4. Finlayson, G.D., Drew, M.S., Funt, B.V.: Diagonal transforms suffice for color constancy. In: Proc. of International Conference of Computer Vision (1993)

5. Finlayson, G.D., Drew, M.S., Funt, B.V.: Spectral sharpening: sensor transformations for improved color constancy. J. Opt. Soc. Am. A 11(5), 1553-1563 (1994)

6. Finlayson, G.D., Hordley, S.D., Hubel, P.M.: Color by correlation: a simple, unifying framework for color constancy. IEEE Transactions on Pattern Analysis and Machine Intelligence 23(11), 1209-1221 (2001)

7. Finlayson, G.D., Hordley, S.D., Lu, C., Drew, M.S.: On the removal of shadows from images. IEEE Trans. Pattern Anal. Mach. Intell. 28, 59-68 (2006)

8. He, Q., Chu, C.-h.H.: Intrinsic images by fisher linear discriminant. In: Bebis, G., Boyle, R., Parvin, B., Koracin, D., Paragios, N., Tanveer, S.-M., Ju, T., Liu, Z., Coquillart, S., Cruz-Neira, C., Müller, T., Malzbender, T. (eds.) ISVC 2007, Part II. LNCS, vol. 4842, pp. 349-356. Springer, Heidelberg (2007)

9. Judd, D.B., MacAdam, D.L., Wyszecki, G., Budde, H.W., Condit, H.R., Henderson, S.T., Simonds, J.L.: Spectral distribution of typical daylight as a function of correlated color temperature. J. Opt. Soc. Am. 54(8), 1031-1036 (1964)

10. Kelly, K.L.: Lines of constant correlated color temperature based on macadam's $(u, v)$ uniform chromaticity transformation of the cie diagram. J. Opt. Soc. Am. 53(8), 999-1002 (1963)

11. Lam, K.M.: Metamerism and colour constancy (1985)

12. Lecca, M., Messelodi, S.: Computing von kries illuminant changes by piecewise inversion of cumulative color histograms. ELCVIA 8(2) (2009)

13. Lecca, M., Messelodi, S.: Illuminant Change Estimation via Minimization of Color Histogram Divergence. In: Trémeau, A., Schettini, R., Tominaga, S. (eds.) CCIW 2009. LNCS, vol. 5646, pp. 41-50. Springer, Heidelberg (2009)

14. McCamy, C.S.: Correlated color temperature as an explicit function of chromaticity coordinates. Color Research \& Application 17(2), 142-144 (1992)

15. Ojala, T., Topi, M., Pietikäinen, M., Viertola, J., Kyllönen, J., Huovinen, S.: Outex - new framework for empirical evaluation of texture analysis algorithms. In: Proc. of the 16 th ICPR 2002, vol. 1, p. 10701. IEEE Computer Society, Los Alamitos (2002)

16. Ozden, M., Polat, E.: A color image segmentation approach for content-based image retrieval. Pattern Recognition, 1318-1325 (2007)

17. Reinhard, E., Ashikhmin, M., Gooch, B., Shirley, P.: Color transfer between images. IEEE Comput. Graph. Appl. 21(5), 34-41 (2001)

18. Schettini, R., Ciocca, G., Zuffi, S.: A survey of methods for colour image indexing and retrieval in image databases. In: Color Imaging Science: Exploiting Digital Media (2001)

19. Tominaga, S., Ishida, A., Wandell, B.A.: Color temperature estimation of scene illumination by the sensor correlation method. Syst. Comput. Japan 38, 95-108 (2007)

20. Takahama, K., Nayatani, Y., Sobagaki, H.: Formulation of a nonlinear model of chromatic adaptation. Color Research \& Application 6(3) (1981) 